\title{
Plasmonics of Supported Nanoparticles Reveals Adhesion at the Nanoscale: Implications for Metals on Dielectrics
}

\author{
Rémi Lazzari,* Jacques Jupille, Rémi Cavallotti, Ekaterina Chernysheva, Sebastián Castilla, \\ Maya Messaykeh, Quentin Hérault, Iryna Gozhyk, and Elisa Meriggio
}

Cite This: ACS Appl. Nano Mater. 2020, 3, 12157-12168

Read Online

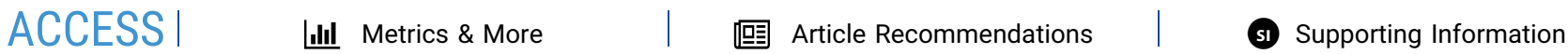

ABSTRACT: The morphology and adhesion energy of nanosized metal particles supported on dielectrics are a puzzling issue since, due to the increasing contribution of surfaces and interfaces in their energetics, their equilibrium shape escapes the rules established for large objects. The evolution of wetting during Volmer-Weber growth of nanoparticles is herein studied by in situ ultraviolet/visible surface differential reflectivity spectroscopy (SDRS). The integrated s-polarized SDR signal is shown to be proportional to the oscillator strength of the optically excited plasmon resonances parallel to the surface. Dielectric modelings show that this quantity, which is marginally affected by the size and density of the

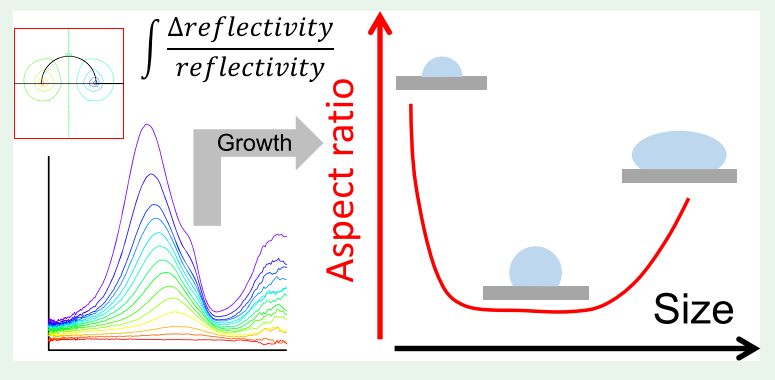
objects, depends mainly on the aspect ratio of the particles from which adhesion energy can be derived. Applied to noble (Ag, Au) or transition metals $(\mathrm{Cr}, \mathrm{Ni})$ and $\mathrm{Zn}$ on weakly interacting dielectric $\left(\mathrm{Al}_{2} \mathrm{O}_{3}, \mathrm{SiO}_{2}, \mathrm{KBr}\right)$ and semiconducting $(\mathrm{TiO}, \mathrm{ZnO})$ substrates, this plasmonic approach evidences a robust U-shaped variation of the aspect ratio with film thickness and therefore size. In line with the thorough study of the $\mathrm{Ag} / \mathrm{Al}_{2} \mathrm{O}_{3}(0001)$ growth and linear elasticity predictions of the equilibrium shape of strained epitaxial particles, the first branch of the " $U$ " is assigned to a size-dependent equilibrium shape related to surface/interface stress effects. A significant decrease in adhesion energy parallels a rounding of the particles. The second branch partly stems from flattening due to incomplete coalescence. The common behavior of poorly wetting supported metal nanoparticles that is revealed herein, with strong changes in shape and adhesion as a function of particle size, had not been evidenced so far. Both the proposed optical methodology and the final findings about adhesion at the nanoscale are of interest in the wide field of application of supported metal nanoparticles that involves heterogeneous catalysis and thin film growth.

KEYWORDS: adhesion, plasmonics, metallic nanoparticles, Wulff-Kaischew-Winterbottom shape, metal/dielectric, growth, differential reactivity, truncated sphere and spheroid

\section{INTRODUCTION}

Wetting at metal/dielectric interfaces ${ }^{1}$ is a common concern in many different interface configurations, such as dispersed particles of controlled sizes in heterogeneous catalysis, ${ }^{2,3}$ buried interfaces in welding/bonding, ${ }^{4,5}$ and continuous films in coatings. ${ }^{6}$ Its understanding, and thereby its control, is crucial for the development of structures with defined properties and of stable morphologies, capable to resist aging under their conditions of use. ${ }^{6,7}$ The investigation of energetics of interfaces has motivated a considerable research effort dedicated to both the thermodynamic and the kinetic aspects of the growth of supported metal films. ${ }^{1,2,6,8-11}$ The poor wetting of dielectric substrates by metals leads to the formation of three-dimensional particles on the surface that follows the so-called Volmer-Weber growth mode. ${ }^{12}$ The geometry at equilibrium reveals the energetics of the system through the Young-Dupré equation of wetting or its Wulff-KaischewWinterbottom (WKW) counterpart for anisotropic surface energies. ${ }^{1,9,13-15}$ Well defined for melted metals and macroscopic-sized supported crystallites, ${ }^{1,13,14}$ the profile of particles is increasingly affected by the surface and interface stresses when the size decreases to enter the nanometric domain. ${ }^{9,11,15,16}$ Moreover, on crystalline substrates, the linear elasticity theory of equilibrium shape ${ }^{9,15}$ and atomistic simulations ${ }^{17-19}$ showed that epitaxial strain always acts against wetting.

The effects become dramatic for dimensions less than around $10 \mathrm{~nm}$, where strong changes in shape and adhesion occur, ${ }^{19-21}$ while in parallel, the particle energetics, which escapes the models that rule the macroscopic scale of extended surfaces, ${ }^{7}$ is not settled yet. The case is of paramount importance in domains, such as catalysis, for which nanoparticles are the active form of the matter. ${ }^{2,8}$ On the one hand,

Received: October 2, 2020

Accepted: November 30, 2020

Published: December 10, 2020 
Volmer-Weber growth mode

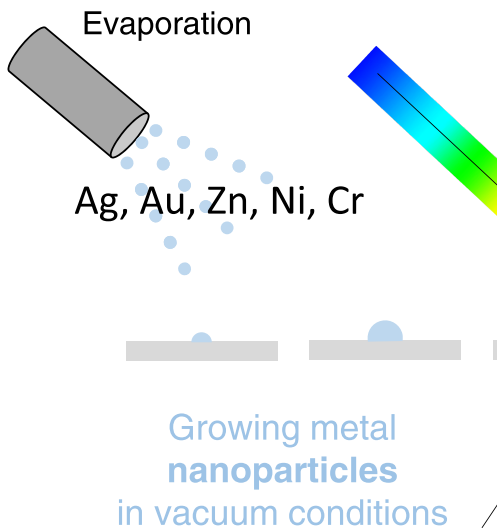

In situ in real time UV-visible
reflectivity UV-visible
reflectivity reflectivity

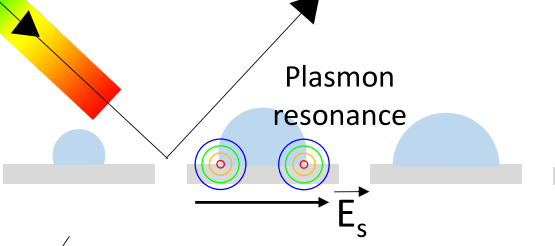
Dielectric substrates
Universal behavior for metal/dielectrics adhesion $=f($ size $)$

via

the determination of the aspect ratio shown to be proportional to the integrated s-polarized light

Figure 1. Scheme of the methodology used to determine the aspect ratio of growing metallic nanoparticles supported on dielectric substrates, from which the adhesion energy is determined, through a dielectric modeling of the plasmonic response probed by UV-visible reflectivity.

in the absence of direct probe at the nanoscale, the relative contributions of surface and interface energies and stresses, which are possibly size-dependent, are still debated (ref 19 and references therein). On the other hand, particle shapes are obviously also altered by kinetics. The insufficient surface diffusion can promote the so-called elongation transition, the formation of worm-like objects and finally the film percolation. $^{6,22-25}$ Thus, continuous metal/dielectric films commonly form through an out-of-equilibrium process so that they are prone to dewetting. ${ }^{1,6,26,27}$ To define the behavior of adhesion and wetting of supported nanoparticles, the challenge is to directly determine the morphology of nanoparticles during their growth.

Of invaluable help to determine the shape of particles, the microscopies are not always well suited to study nanometerscale growing aggregates. They must be operated in situ or handled under special conditions to avoid oxidation, change of shape, and sintering upon air exposure. Time-consuming electron microscopy ${ }^{2}$ requires, beyond specific sample preparation, in-plane and transverse views, while near-field techniques suffer from tip convolution. Conversely, light-based tools provide direct methods to probe growth processes on surfaces in real time. Grazing incidence small-angle X-ray scattering (GISAXS) ${ }^{28,29}$ allows for a detailed description of phenomena in reciprocal space but requires the use of a synchrotron source with all related drawbacks. Yet, the growth of metals on dielectrics can be conveniently monitored by laboratory ultraviolet(UV)/visible spectroscopy through the well-established sensitivity of plasmon resonances to particle size and shape. ${ }^{30-33}$ In this context, surface differential reflectivity spectroscopy (SDRS) has already shown its capability for the quantitative study of the growth mode and adhesion of metals on oxides as for $\mathrm{Ag}^{19,20,34} \mathrm{Al}^{34}{ }^{34} \mathrm{Ti}^{34}$ and $\mathrm{Zn}^{35}$ on $\mathrm{Al}_{2} \mathrm{O}_{3}(0001)$ and $\mathrm{Ag}$ on $\mathrm{MgO}(100)^{36}$ that fairly compares to GISAXS analysis. Being quite flexible, SDRS was also applied to the in situ growth analysis during deposition via plasma sputtering. ${ }^{24,37}$ In fact, plasmonic absorptions are driven to a great extent by the aspect ratio of the particle, defined as the ratio of the in-plane diameter divided by the emerging height. However, the direct reading of that parameter is blurred by the excitations of several shape-related polarization modes, by polydispersity or finite-size broadenings, and by strong dielectric damping in transition metals. ${ }^{38,39}$ Therefore, a specific modeling is required to derive the nanoparticle morphology from the spectral line shape. It must describe nanoparticles of realistic shapes (truncated spheres ${ }^{40-42}$ or spheroids ${ }^{43}$ ) in interaction with the substrate (image effects) and other particles ${ }^{44}$ in polydisperse assemblies and account for finite-size and temperature effects on the dielectric constant of the particle material. ${ }^{45}$ Such an approach must clearly be adapted to each particular system under study.

We propose herein to follow a different strategy to develop a qualitative but widely applicable method for monitoring the evolution of wetting during the growth of supported nanoparticles by vapor deposition (Figure 1). It does not rely on the complete analysis of line shapes but on integrated signal in s-polarization, which, once normalized to average film thickness, is shown to be proportional to the aspect ratio of the nanoparticles and at the same time independent of their density and size. The methodology that relies on real-time in situ measurements is applied to various metal/dielectric systems during their growth. It enables us to derive trends in the variation of adhesion energy and allows us to estimate this energy through the Young-Dupré equation. ${ }^{1}$

\section{EXPERIMENTAL SECTION}

The film growths have been performed in ultra-high vacuum vessels (base pressure in the high $10^{-11} \mathrm{mbar}$ ) by thermal evaporation from carefully out-gassed crucibles $(\mathrm{Ag}, \mathrm{Au}, \mathrm{Zn}, \mathrm{Ni})$ or by electron bombardment of a metallic rod ( $\mathrm{Cr}$ ) (Omicron EFM3 evaporator). The growth was monitored in situ by surface differential reflectivity spectroscopy (SDRS) in the UV-visible range $(\sim 1.5-5 \mathrm{eV})$ with a setup described elsewhere. ${ }^{39}$ The incident angle was fixed to $\theta_{0}=45^{\circ}$ or to $55^{\circ}$ by the chamber flanges equipped with transparent silica viewports. Prior to deposition, the evaporation rate of the order of 0.1 $\mathrm{nm} / \mathrm{min}$ was systematically calibrated by a quartz balance set at the sample position. If not stated, the sample was held at room temperature during growth. The surfaces of single crystal substrates were prepared either by (i) annealing under a partial pressure of oxygen provided by a gas doser set in front of the surface $(\sim 1200 \mathrm{~K}$; stationary pressure of $10^{-6}$ mbar) for $\mathrm{Al}_{2} \mathrm{O}_{3}(0001)$ and amorphous $\mathrm{SiO}_{2}$ or (ii) by cycles of sputtering/annealing $\left(\mathrm{Ar}^{+} ; 1 \mathrm{keV} ; \sim 1100 \mathrm{~K}\right)$ for $\mathrm{ZnO}(0001)-\mathrm{Zn}$ and $\mathrm{TiO}_{2}(110)$ or (iii) by cleavage in air followed by slight degassing in vacuum for $\mathrm{KBr}(001)$. The surface preparation allowed to reduce the amount of surface contaminants below the sensitivity of X-ray photoemission spectroscopy and led to sharp and 

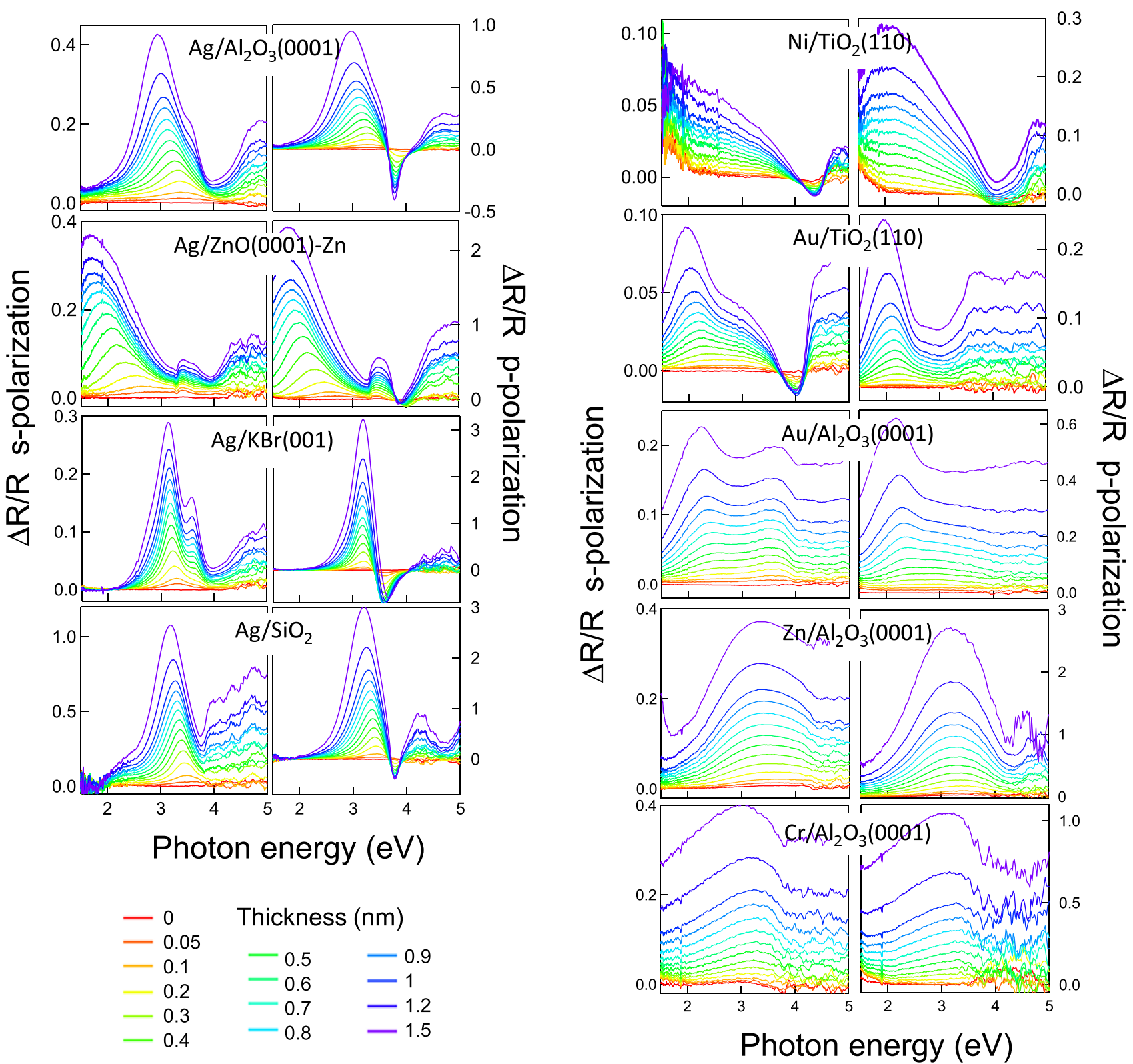

Figure 2. Surface differential reflectivity spectra acquired with s-polarized (left) and p-polarized (right) light during the growth of metal nanoparticles on a substrate as indicated in each subfigure. Average film thickness in $\mathrm{nm}$, as calibrated by a quartz balance, are indicated by color codes given in the legend. The evaporation rate was of the order of $0.1 \mathrm{~nm} / \mathrm{min}$ for all systems. All growths are performed at room temperature, except for $\mathrm{Zn} / \mathrm{Al}_{2} \mathrm{O}_{3}(0001)$, which requires cooling around $100 \mathrm{~K}$ to achieve a sizable sticking coefficient. ${ }^{35}$

decent $(1 \times 1)$ low-energy electron diffraction patterns for single crystals.

Briefly, in SDRS, the growth of a metallic layer is monitored through the relative variation of the sample reflectivity simultaneously in its s- and p-polarized components. It might be worth reminding that, under p-polarized illumination, the electric field lies within the plane of incidence and can be decomposed into two components, one parallel and another perpendicular to the substrate surface. However, only the electric field component parallel to the substrate is present under s-polarized illumination. The measured SDRS s- or p-polarized signal is given by

$$
\frac{\Delta R}{R}(\omega, t)=\frac{R(\omega, t)-R(\omega, 0)}{R(\omega, 0)}
$$

where $\omega / 2 \pi$ is the photon frequency, $R(\omega, 0)$ is the reflection coefficient of the bare substrate and $R(\omega, t)$ that of the surface covered by a deposit of thickness $t$. The normalization to $R(\omega, t=0)$ is necessary to cancel out experimentally, for each value of $\omega$, the unknown response of the optical bench, i.e., the lamp emission, the transmission of the optics, the monochromator transfer function, and the detector response. However, it is of paramount importance to have a stable optical bench throughout the measurements to assume these quantities as constants. ${ }^{39}$

\section{RESULTS AND DISCUSSION}

3.1. Differential Reflectivity Spectra from Growing Metal/Dielectric Films. SDR spectra were collected in situ during the growth of $\mathrm{Ag}, \mathrm{Au}, \mathrm{Cr}, \mathrm{Zn} / \mathrm{Al}_{2} \mathrm{O}_{3}(0001),{ }^{19,35,39,46}$ $\mathrm{Ag} / \mathrm{KBr}(001), \mathrm{Ag} / \mathrm{ZnO}(0001)-\mathrm{Zn}, \mathrm{Au} / \mathrm{TiO}_{2}(110),{ }^{39} \mathrm{Ni} /$ $\mathrm{TiO}_{2}(110)$, and $\mathrm{Ag} / \mathrm{SiO}_{2}$ (Figure 2). As indicated by the provided citations, some of these interfaces have already been studied by the authors but never in the present context. The optical response of a metal deposit on a dielectric substrate is 
quite sensitive to the variation of the amount of metal, generally described through the mean film thickness. A submonolayer sensitivity is easily achieved due to the high contrast between the dielectric constants of the two media and the low absolute reflection coefficient especially in p-polarization near the Brewster angle of the substrate. For silver, whose surface plasmon resonance is the sharpest in the UVvisible range, SDR spectra can be recorded for mean film thicknesses smaller than a tenth of a monolayer, i.e., 0.015 $\mathrm{nm} .^{20}$ The smallest thickness analyzed here is $0.05 \mathrm{~nm}$, which is the limit of signal-to-noise ratio for signal integration for some of the films under study.

Noble metals commonly used in plasmonics, such as Ag and $\mathrm{Au}$, are herein compared to $\mathrm{Zn}$ and transition metals $\mathrm{Cr}$ and $\mathrm{Ni}$, in which the band-to-band transitions play a non-negligible role. ${ }^{39,47}$ Plasmonic properties of metals degrade when free and bound electrons (or in other words, intraband and interband contributions) get mixed in the resonant modes. In this regard, $\operatorname{Ag}\left(4 d^{10} 5 s^{1}\right), \operatorname{Au}\left(5 d^{10} 6 s^{1}\right), \operatorname{Zn}\left(3 d^{10} 4 s^{1}\right), \operatorname{Cr}\left(3 d^{5} 4 s^{1}\right)$, and $\mathrm{Ni}-$ $\left(3 d^{9} 4 s^{1}\right)$ have similar s-shell filled with one electron but different d-shell fillings. Despite similar Drude parameters, ${ }^{39}$ they differ drastically on their interband transitions that progressively shift from 4 to $1 \mathrm{eV}$ from $\mathrm{Ag}, \mathrm{Au}, \mathrm{Zn}$, to $\mathrm{Cr}$ and $\mathrm{Ni}$ (Figure S1 of the Supporting Information). These five metals are quite representative of various dielectric configurations of screening of s electrons by transitions involving $d$ electrons. In silver, plasmon resonances occur in the energy range well below that of the interband transitions. In gold, they are closer to interband transitions while in $\mathrm{Zn}, \mathrm{Cr}$, and $\mathrm{Ni}$, they are expected to happen on the tail of the interband transitions leading to a more complex line shape of plasmon resonances. ${ }^{39}$

As for the analyzed substrates, they are weakly interacting with the metal deposit. $\mathrm{Al}_{2} \mathrm{O}_{3}, \mathrm{SiO}_{2}$, and $\mathrm{KBr}$ are archetypes of wide band gap non-absorbing substrates, either crystalline with different symmetries or amorphous, with a nearly constant dielectric function in the UV-visible range. On the other hand, $\mathrm{ZnO}$ and $\mathrm{TiO}_{2}$ are semiconductors with a band gap in the middle of the probed range (Figure S2).

3.2. Fresnel Reflection Coefficients in the LongWavelength Approximation. The macroscopic optical response of a film of supported nanoparticles can be handled as that of a transition interface between two homogeneous media, ${ }^{44,48-52}$ provided that nanoparticle sizes and interparticle distances are much smaller than the optical wavelength $\lambda$. This generalization of the thin plate model ${ }^{53}$ relies on the concept of excess electromagnetic fields, which are the difference between the actual fields and those of the homogeneous media extrapolated to the surface. ${ }^{44}$ The boundary conditions at such a fictitious interface are obtained by integrating the excess electromagnetic fields perpendicularly to the surface. These unknown integrals are linked to the bulk fields extrapolated to the surface through interface susceptibilities $\gamma(\omega)$ and $\beta(\omega)$. Those are frequency-dependent dielectric lengths that describe the interface polarization along the directions parallel $(\gamma)$ and perpendicular $(\beta)$ to it. $^{44,48-52}$ The linear Fresnel coefficients in amplitude ${ }^{44,48,52}$ can be derived for such an interface from the Maxwell equations. Greatly simplified expressions of the differential reflectivity signals are obtained in the framework of the long-wavelength approximation, ${ }^{44,45,48}$ i.e., $2 \pi|\gamma| \ll \lambda$ and $2 \pi|\beta| \ll \lambda$, in which interface susceptibilities are small with respect to the light wavelength. In addition to the abovementioned smallness of the particle size with respect to light wavelength, this requires a moderate resonant behavior for the interface susceptibilities. ${ }^{47}$ In this regime, the differential reflectivity signals in $\mathrm{s}$ and $\mathrm{p}$ polarizations read $^{47}$

$$
\begin{aligned}
\frac{\Delta R_{s}}{R_{s}}= & \frac{8 \pi}{\lambda} \frac{n_{a} \cos \theta_{0}}{\epsilon_{s}-\epsilon_{a}} \operatorname{Im}[\gamma] \\
\frac{\Delta R_{p}}{R_{p}}= & \frac{8 \pi}{\lambda} \frac{n_{a} \cos \theta_{0}}{\left(\epsilon_{s}-\epsilon_{a}\right)\left(\epsilon_{s} \cos ^{2} \theta_{0}-\epsilon_{a} \sin ^{2} \theta_{0}\right)} \\
& \times\left[\left(\epsilon_{s}-\epsilon_{a} \sin ^{2} \theta_{0}\right) \operatorname{Im}(\gamma)-\epsilon_{s}^{2} \epsilon_{a} \sin ^{2} \theta_{0} \operatorname{Im}(\beta)\right]
\end{aligned}
$$

for a non-absorbing substrate under illumination at the incident angle $\theta_{0} . \epsilon_{a}$ and $\epsilon_{s}$ are the incoming medium (vacuum herein) and substrate dielectric functions, respectively, and $n_{a}=\sqrt{\epsilon_{a}}$ is the index of refraction. Frequency dependence of quantities is implicit in the previous equations, and if not stated, in what follows.

For supported nanoparticles ${ }^{44}$

$$
\gamma(\omega)=\rho\left\langle\alpha_{\|}(\omega)\right\rangle \text { and } \beta(\omega)=\rho\left\langle\alpha_{\perp}(\omega)\right\rangle / \epsilon_{a}^{2}
$$

where $\rho$ is the particle density and $\left\langle\alpha_{\|}(\omega)\right\rangle$ and $\left\langle\alpha_{\perp}(\omega)\right\rangle$ are the parallel and perpendicular components, respectively, of the average tensor of polarizability of the particles, which links the induced damped dipole in each particle to the excitation electric field of the incident light. ${ }^{44}$ The polarizabilities $\alpha_{\|}$and $\alpha_{\perp}$ can be calculated by solving the Laplace equation for specific shapes in the so-called quasi-static approximation, i.e., by neglecting retardation effects, since particles are small with respect to the light wavelength. ${ }^{40-44,54}$ Note that, since the polarizabilities scale with particle volume $V$, the interface susceptibilities are proportional to the average film thickness:

$$
t=\rho\langle V\rangle
$$

In the systems under study, films are a few monolayers thick and well below the percolation threshold; the size of metal/ dielectric particles happens to be small enough to fulfill the long-wavelength assumption despite the plasmonic resonant behavior (see ref 47). For instance, at the maximum thickness of $1.5 \mathrm{~nm}$ used herein (Figure 2), SDRS and GISAXS show that oxide-supported silver particles are around $5 \mathrm{~nm}$ in size, ${ }^{20,36,45}$ which corresponds to a few percent of the wavelengths used to record the present spectra.

The above equations (eqs 2 and 3 ) account for some of the main features of the experimental line shapes of Figure 2. The s-polarized spectra only depend on the polarization in the direction parallel to the surface ( $\gamma$ term in eq 2). In contrast, the p-polarized signal involves a complex subtraction of terms proportional to $\operatorname{Im}[\gamma(\omega)]$ and $\operatorname{Im}[\beta(\omega)]$ (eq 3). Although not obviously featured in spectra related to metal involving bandto-band transitions such as $\mathrm{Au}, \mathrm{Zn}, \mathrm{Cr}$, and $\mathrm{Ni}$ (Figure 2), the two contributions directly appear in the p-polarized spectra of $\mathrm{Ag}$ on $\mathrm{Al}_{2} \mathrm{O}_{3}(0001), \mathrm{KBr}(001), \mathrm{ZnO}(0001)-\mathrm{Zn}$, and $\mathrm{SiO}_{2}$ (Figure 2). The sharp dips seen ca. $3.5-4 \mathrm{eV}$ stem from the absorption modes perpendicular to the substrate. Conversely, broad bands peaking below $3.5 \mathrm{eV}$ due to modes parallel to the substrate dominate s-polarized spectra. ${ }^{38,39}$ The quantitative analysis of $\mathrm{Ag}$ growth and adhesion has been successfully achieved on various surfaces thanks to this combination of modes. ${ }^{19,20,24,34,36}$ In contrast, in the qualitative approach developed herein for all metals, the spolarized reflectivity spectra are shown below to be the most 
relevant for a direct determination of the aspect ratio of growing metal particles.

3.3. Oscillator Strengths of Plasmon Resonances. The plasmonics of nanoparticles is dictated by their polarizabilities that behave mostly as oscillators. This section evidences the links of the corresponding frequencies and strengths to the particle aspect ratio via the development of appropriate particle models starting from freestanding objects and gradually approaching to interacting supported particles of realistic shapes.

A tractable and illustrative starting point to represent particles is the textbook freestanding full spheroid, oblate, or prolate $^{32,33,44}$ (Figure 3, top) embedded in a homogeneous

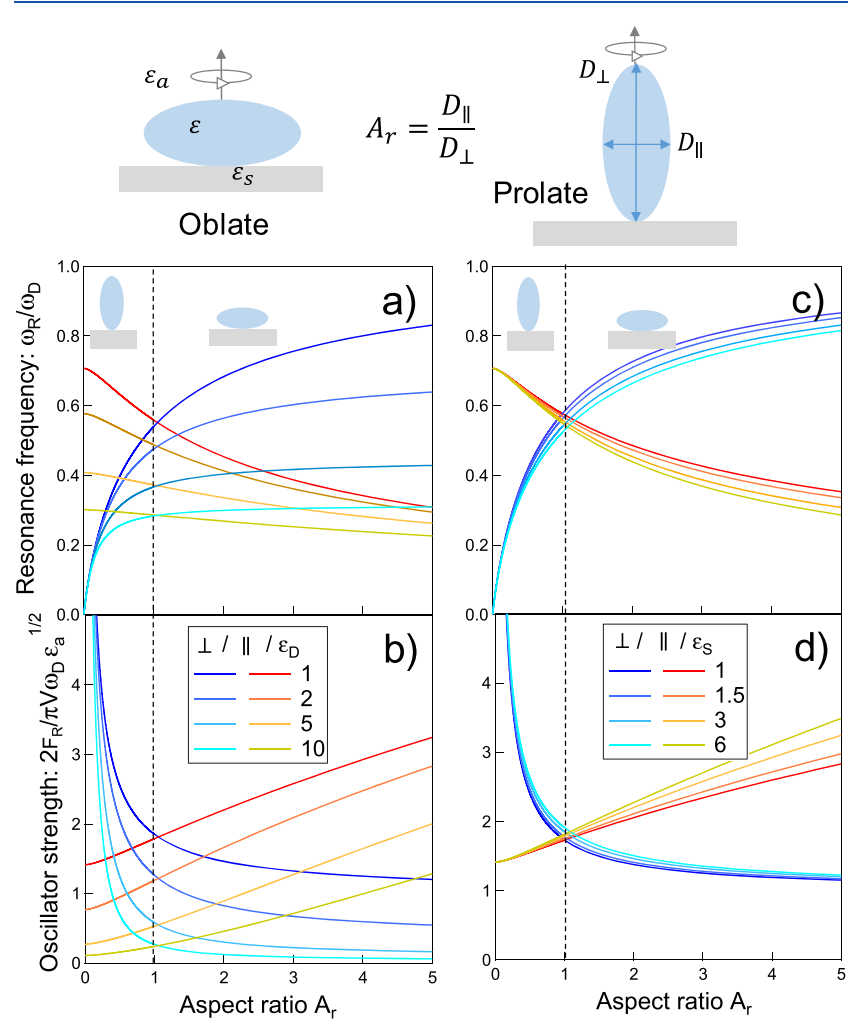

Figure 3. Oscillator behavior of supported full spheroids determined in the framework of the SDA approximation. Top: schematic representation of the prolate and oblate spheroids that features dielectric functions and particle aspect ratio (see text). Bottom: the particle dielectric function is described by a Drude term with either $(\mathrm{a}, \mathrm{b})$ a variable (indicated in figure) or (c, d) a constant residual term $\varepsilon_{D}=1$. The dielectric constant of the substrate is either $(\mathrm{a}, \mathrm{b})$ constant $\varepsilon_{s}=3$ or $(\mathrm{c}, \mathrm{d})$ variable as indicated in the legend. Oscillator characteristics: (a, c) normalized resonance frequency $\omega_{R} / \omega_{D}$ and (b, d) normalized oscillator strength $2 F_{R} / \pi V \omega_{D} \sqrt{\varepsilon_{a}}$ of a prolate/oblate spheroid (see Section SII.1 for definitions). The dashed line corresponds to the spherical shape $\left(A_{r}=1\right)$.

medium. Its polarizability tensor is dominated by dipolar modes along the directions parallel or perpendicular to its rotation axis: ${ }^{32,33,44}$

$$
\alpha_{\|, \perp}(\omega)=\frac{\varepsilon_{a}\left(\varepsilon-\varepsilon_{a}\right) V}{\varepsilon_{a}+L_{\|, \perp}\left(\varepsilon-\varepsilon_{a}\right)}
$$

where $\varepsilon$ (a function of $\omega$, even if omitted hereafter) is the dielectric function of the particle material and $\varepsilon_{a}$ that of the ambient medium. For plasmonic media with low absorption, a resonance is found when $\varepsilon_{a}+L_{\|, \perp}\left(\varepsilon-\varepsilon_{a}\right)=0$ or, in other words, for a particle dielectric function $\varepsilon_{R}=\varepsilon_{a}\left(1-\frac{1}{L}\right)$. The depolarization factors $L=L_{\|, \perp}$ (Section SII.2) depend on the aspect ratio $A_{r}$ of the particle, ${ }^{44,54}$ defined as the ratio of dimensions parallel and perpendicular to the rotation axis $A_{r}=$ $D_{\|} / D_{\perp}$ (Figure 3, top). For a Drude metal with dielectric function $\varepsilon(\omega)=\varepsilon_{D}-\omega_{D}^{2} /\left(\omega^{2}+i \omega \Gamma_{D}\right)$, a plasma frequency $\omega_{D}$ and a damping $\Gamma_{D}$, polarizabilities can be expressed in the form of a damped oscillator (Section SII.1):

$$
\alpha(\omega)=\frac{2}{\pi} \frac{\omega_{R} F_{R}}{\omega_{R}^{2}-\omega^{2}-i \omega \Gamma_{D}}
$$

when $\omega$ is close to its resonant frequency $\omega_{R}$ and when $\Gamma_{D} \ll$ $\omega_{R}<\omega_{D}$. Both the oscillator strength $F_{R}$ and the resonant frequency $\omega_{R}$ are functions of $L=L_{\|, \perp}$. Their values normalized to the Drude frequency $\frac{\omega_{R}}{\omega_{D}}$ and $\frac{2 F_{R}}{\pi V \omega_{D} \sqrt{\varepsilon_{a}}}$ are given in Section SII.1.

Now, placed in front of a substrate, such spheroid induces an image polarization. In the simple dipole picture, eq 7 remains valid but with depolarization factors that depend on the image strength $\frac{\varepsilon_{s}-\varepsilon_{a} 44,45,54}{\varepsilon_{s}+\varepsilon_{a}}\left(\varepsilon_{a}\right.$ and $\varepsilon_{s}$ stand for the vacuum and substrate dielectric functions, respectively) and on the distance $d$ between the surface and the center of spheroid. Appropriate expressions of $L_{\|, \perp}$ can be obtained in the framework of the spheroid dipole approximation (SDA $)^{44,54}$ by restricting to dipolar order the multipolar solution of the Laplace equation $^{44,45,54}$ (Section SII.2). For a Drude metal, the normalized oscillator frequencies (Eq S4) and strengths (Eq S5) are shown in Figure 3 as a function of the aspect ratio. To represent a variety of cases, modeling uses (i) either a variable residual term $\varepsilon_{D}$ at fixed substrate dielectric function $\varepsilon_{s}=3$ (Figure $3 \mathrm{a}, \mathrm{b}$ ) or (ii) a variable $\varepsilon_{s}$, i.e., variable image strength, at fixed $\varepsilon_{D}=1$ (Figure $3 \mathrm{c}, \mathrm{d}$ ). The well-known red (blue) shift of the resonance parallel (perpendicular) to the surface upon particle flattening is reproduced. Most importantly, the evolution of the oscillator strength as a function of the aspect ratio $A_{r}$, which is complex in the direction perpendicular to the substrate, is nearly linear along the parallel direction (Figure $3 \mathrm{~b}, \mathrm{~d})$. This linear increase in $F_{R, \|}\left(A_{r}\right)$ is at the heart of the analysis proposed below and will be shown to be robust for different system configurations.

The next step is to consider supported particle assemblies, i.e., the modification by neighbors of the local field felt by each particle $44,54,55$ (Section SIII). The particle-particle electrostatic coupling at dipolar order was proven to be satisfactory with respect to quadrupolar order for surface coverage lower than $0.5 .^{56,57}$ This dipolar coupling leads to a renormalization of the polarizabilities and new expressions for the depolarization factors (see Section SIII). Moreover, at such surface coverage, no significant difference was observed between optical properties of random, square, or hexagonal lattice geometries. ${ }^{56}$ For the sake of simplicity, a square lattice of parameter $P$, whose surface coverage is defined as $\Theta=\pi D_{\|}^{2} /$ $4 P^{2}$, is used hereafter. As expected, particle-particle coupling increases the splitting of frequencies between resonances parallel (red-shifted) and perpendicular (blue-shifted) to the surface (Figure 4). In the realistic case of oblate spheroids, the linear increase in $F_{R, \|}$ with $A_{r}$ highlighted for isolated particles (Figure 3 ) is again observed (Figure $4 \mathrm{~b}, \mathrm{~d}$ ). In the coverage range $\Theta \lesssim 0.5$, the upper limit of the relative variation of $F_{R, \|}$ 

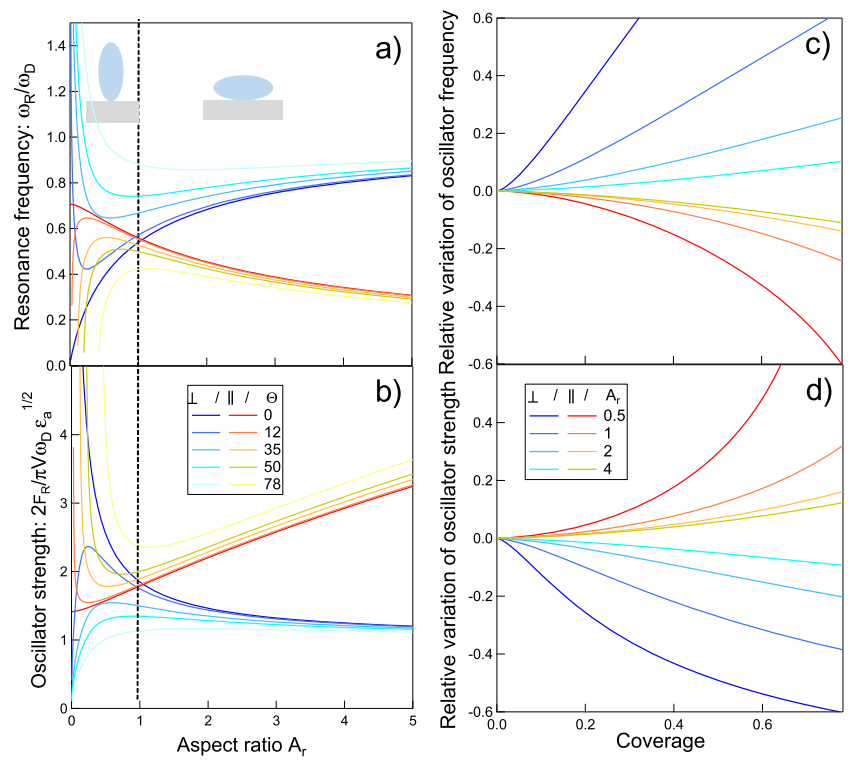

Figure 4. Impact of the aspect ratio and the particle-particle dipolar coupling on the oscillator properties in an assembly of spheroids supported on a substrate with $\varepsilon_{S}=3$ : (a) normalized resonance frequency $\omega_{R} / \omega_{D} ;$ (b) normalized oscillator strength $2 F_{R} / \pi V \omega_{D} \sqrt{\varepsilon_{a}}$; (c, d) their relative variation as a function of $(\mathrm{a}, \mathrm{b})$ aspect ratio $A_{r}$ at fixed coverage $\Theta=\pi D_{\|} / 4 P^{2}$ indicated in the figure or (c, d) vice versa. Spheroids made of Drude metal with $\varepsilon_{D}=1$ are organized in a square lattice of period $P$ (see text); their volume is kept constant while increasing the coverage.

with $\Theta$ is $12 \%$ for a full sphere $\left(A_{r}=1\right)$ and even lower for higher $A_{r}$ values (Figure $4 \mathrm{~d}$ ). Most importantly, the variations of $F_{R, \|}$ and $\omega_{R, \|}$ are mainly driven by the aspect ratio $A_{r}$ for oblate particles, while the coverage appears as a second-order parameter (Figures 3 and 4). Therefore, in the following, the realistic approach to the shape and truncation of supported objects will be developed only around isolated particles.

To describe partial wetting, the optical response of supported nanoparticles is better addressed by modeling these by truncated spheres or spheroids. ${ }^{40,44,58}$ Even if the actual particle shape may be faceted at equilibrium or more complex due to kinetics, the proposed shapes allow to cover a wide range of aspect ratio $A_{r}$, the key parameter in wetting. Moreover, the truncated sphere describes accurately the exact equilibrium shape of a liquid droplet or of a material with a poor surface energy anisotropy such as silver. ${ }^{21} A_{r}$, defined as the ratio of the apparent parallel diameter to the emerging part of the object (Figure 5, top), is not unique to define truncated spheroids since an infinity of such objects correspond to a single value of $A_{r}$. This has led us to define a truncation parameter $t_{r}$ as ratio of the distance from the center of the spheroid to the substrate to the radius of the spheroid in the perpendicular direction $t_{r}=h / R_{\perp}$ (Figure 5, top). Because of the excitation of a wealth of shape-dependent polarization modes due to truncation and image effect, ${ }^{38,39,59-61}$ it was previously shown that polarizabilities of such a system can still be represented in terms of damped oscillators by an expression that generalizes eq 7 (Section SIV):

$$
\alpha(\omega)=\alpha_{b k}(\omega)+\sum_{i} \frac{2}{\pi} \frac{F_{R, i} \omega_{R, i}}{\omega_{R, i}^{2}-\omega^{2}-i \omega \Gamma_{R, i}}
$$

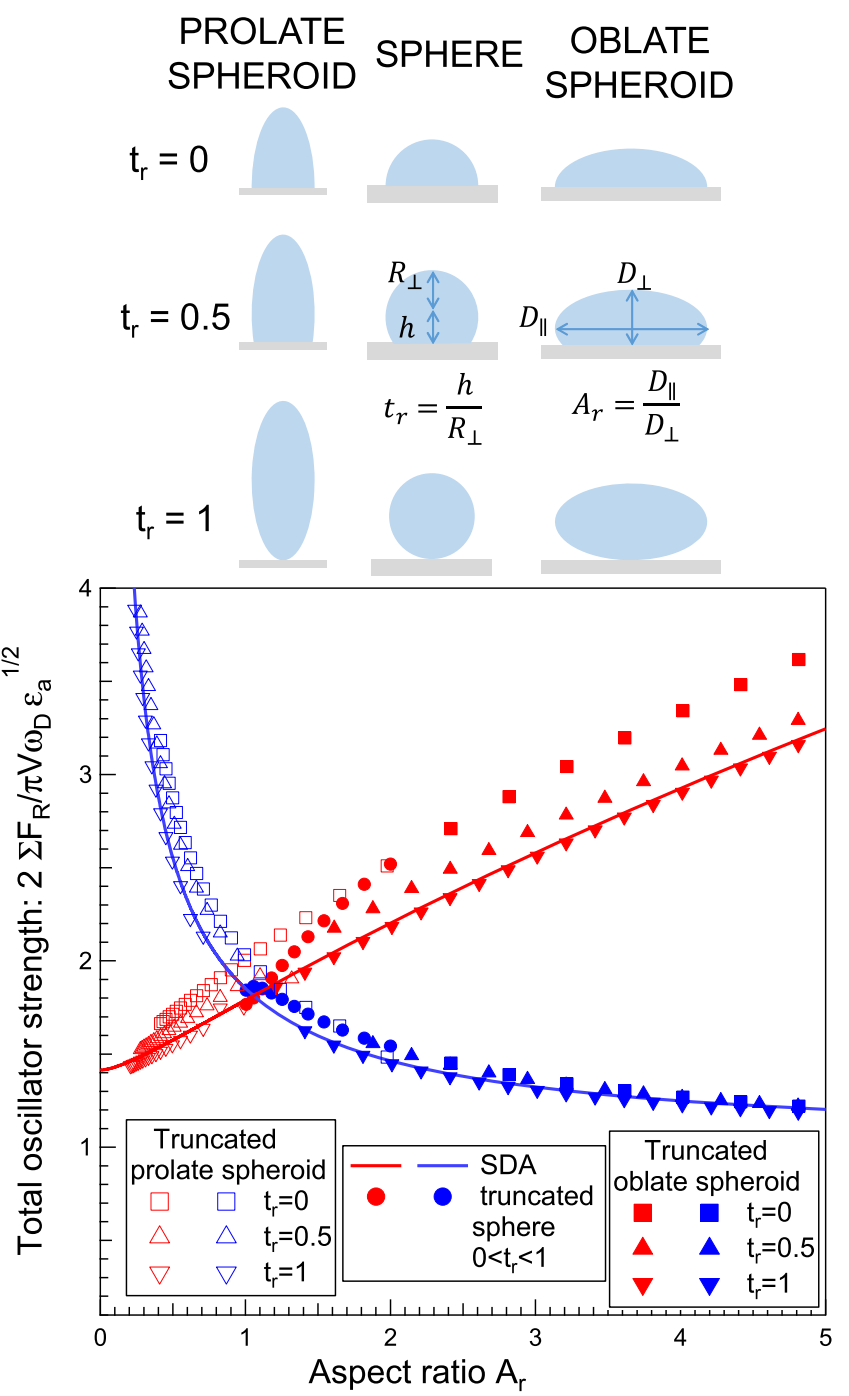

Figure 5. Top: schematic representation of truncated spheroids $\left(t_{r}=\right.$ $\left.h / R_{\perp}\right)$ from hemiparticle $\left(t_{r}=0\right)$ to full particle $\left(t_{r}=1\right)$. Bottom: variation with the particle aspect ratio of the normalized total oscillator strength of truncated spherical (full disks), oblate (full squares and triangles), or prolate (open squares and triangles) spheroidal particles. Blue (red) color corresponds to oscillators oriented in the direction perpendicular (parallel) to the substrate. Calculations are performed at multipolar order $(M=16)$ for $\varepsilon_{a}=1, \varepsilon_{s}$ $=3$, and a Drude metal $\left(\varepsilon_{D}=1\right)$, and for all truncations shown on the top. Results are compared to the SDA model (full lines).

$F_{R, i}, \omega_{R, i}$, and $\Gamma_{R, i}$ are the oscillator strength, the resonance frequency, and the damping of a given polarization mode $i$, respectively. The non-resonant term $\alpha_{b k}(\omega)$, which accounts, in particular, for bulk absorption, is often negligible compared to plasmon oscillations. ${ }^{39}$

Now, to examine whether the linearity of $F_{R, \|}\left(A_{r}\right)$ previously found for full spheroids in SDA at dipolar order (Figure 3) still holds for truncated particles, the exact values of $\operatorname{Im}\left[\alpha_{\|, \perp}(\omega)\right]$ calculated at multipolar order ${ }^{40-44,58}$ for supported truncated spheres/spheroids have been integrated over the full $\omega$ scale for a Drude metal to obtain the normalized-to-volume total oscillator strengths $\Sigma F_{R} / V$ for both parallel and perpendicular directions (eq 8). The integration has been performed for spheroids and spheres of several truncations $t_{r}$ on simulations at multipole order $M=16$ (Figure 5) using the GranFilm software. ${ }^{62,63}$ This value turned out to 
be satisfactory in describing the boundary conditions for the electrostatic potential and for the displacement field at the surface of the object. ${ }^{42}$ Despite the degeneracy of $A_{r}$ with $t_{r}$ and the existence of several modes of polarization, ${ }^{39,60}$ the comparison with the SDA of Figure 5 demonstrates a robust linear dependence of $\Sigma F_{R, \|} / V$ with respect to $A_{r}$. Moreover, calculations made for realistic values of truncation parameter ( 0.5 or 0 ) only slightly differ, which means that the variations of the oscillator strength are mainly driven by change in aspect ratio (Figure 5). In contrast, in line with the case of full spheroids, $\Sigma F_{R, \perp} / V$ shows a non-linear behavior as a function of $A_{r}$ (Figure 5).

Up to now, the analysis was performed for particle and substrate materials described through a textbook Drude dielectric function and a constant value, respectively. Mind that the Drude screening $\varepsilon_{D}$ already impacts significantly the oscillator characteristics (Figure $3 \mathrm{a}, \mathrm{b}$ ). To be closer to real world and to compare with experimental data, $\Sigma F_{R} / V$ has been determined via direct integration of calculated $\operatorname{Im}\left[\alpha_{\|, \perp}(\omega)\right]$ for metal ( $\mathrm{Ag}, \mathrm{Au}, \mathrm{Zn}, \mathrm{Cr}, \mathrm{Ni}) /$ substrate $\left(\mathrm{Al}_{2} \mathrm{O}_{3}, \mathrm{SiO}_{2}, \mathrm{KBr}\right.$, $\mathrm{ZnO}$, and $\mathrm{TiO}_{2}$ ) interfaces by using tabulated dielectric functions $^{64-66}$ (Figures S1 and S2). Only realistic particle shapes in terms of wetting, i.e., with $A_{r}>1$, have been considered in the form of (i) truncated sphere of which the shape is varied from the full sphere $\left(t_{r}=1 ; A_{r}=1\right)$ to hemisphere $\left(t_{r}=0 ; A_{r}=2\right)$ and (ii) hemispheroid $\left(t_{r}=0 ; A_{r}>\right.$ 2). For numerical issues of convergence, ${ }^{42}$ particles with $A_{r}>2$ are better described by truncated spheroids than truncated sphere with $t_{r}<0$. The rather modest impact of the truncation ratio with respect to aspect ratio on the oscillator strengths (Figure 5) allows one to calculate $\Sigma F_{R} / V$ only for a given truncation value $\left(t_{r}=0\right.$ in Figure 5). The integration was restricted to the experimental $\mathrm{UV}-$ visible range $(1.5-5 \mathrm{eV})$. Most importantly, the trend observed in previous sections holds true for actual materials (Figure 6). $\Sigma F_{R, \|} / V$ increases nearly linearly as a function of $A_{r}$ for $A_{r} \gtrsim 1.5$ despite drastically different dielectric functions. In fact, this linearity roots into the dominant role of a parallel "dipole"-like resonant absorption mode in truncated particles, ${ }^{39,60}$ as highlighted in Figure S2 for some selected shapes. Moreover, for realistic aspect ratios $1<A_{r}<6$, the parallel depolarization factor $L_{\|}$ ranges between 0.08 and 0.3 (Figures $S 5$ and $S 6$ ) and the corresponding dielectric function at resonance $\varepsilon_{R}$, $\left(\varepsilon_{R}=\varepsilon_{a}\left(1-\frac{1}{L}\right)\right.$; eq 6) between -11.5 and -2.3 which, for the metals under study (Figure $S 1$ ), falls precisely within the probed UV-visible range. This is a crucial point since the proof that the main "dipolar" resonance is well centered in the probed domain guarantees the reliability of the integration of experimental data involving enlargements induced by polydispersity and finite size. Two particular cases deserve comment. First, although the parallel resonance is expected at two frequencies in $\mathrm{Cr}$ and $\mathrm{Zn}$ (Figure S1), the one at low energy should vanish because of the large imaginary part of the metal dielectric function. ${ }^{39}$ Second, in the case of gold, in contrast with the other metals, the resonances fall onto a positive slope of $\operatorname{Im}(\varepsilon)$ with $\omega$ (low-energy side of the interband transition). This blurs the dominant character of the parallel mode, which is recovered only upon redshift for larger aspect ratios $\left(\mathrm{Au} / \mathrm{Al}_{2} \mathrm{O}_{3}\right.$ in Figure 6) or in the case of substrates with high dielectric function $\left(\mathrm{Au} / \mathrm{TiO}_{2}\right.$ in Figure 6).

The linear relation between the integrated parallel oscillator strength and aspect ratio holds in all the models of supported
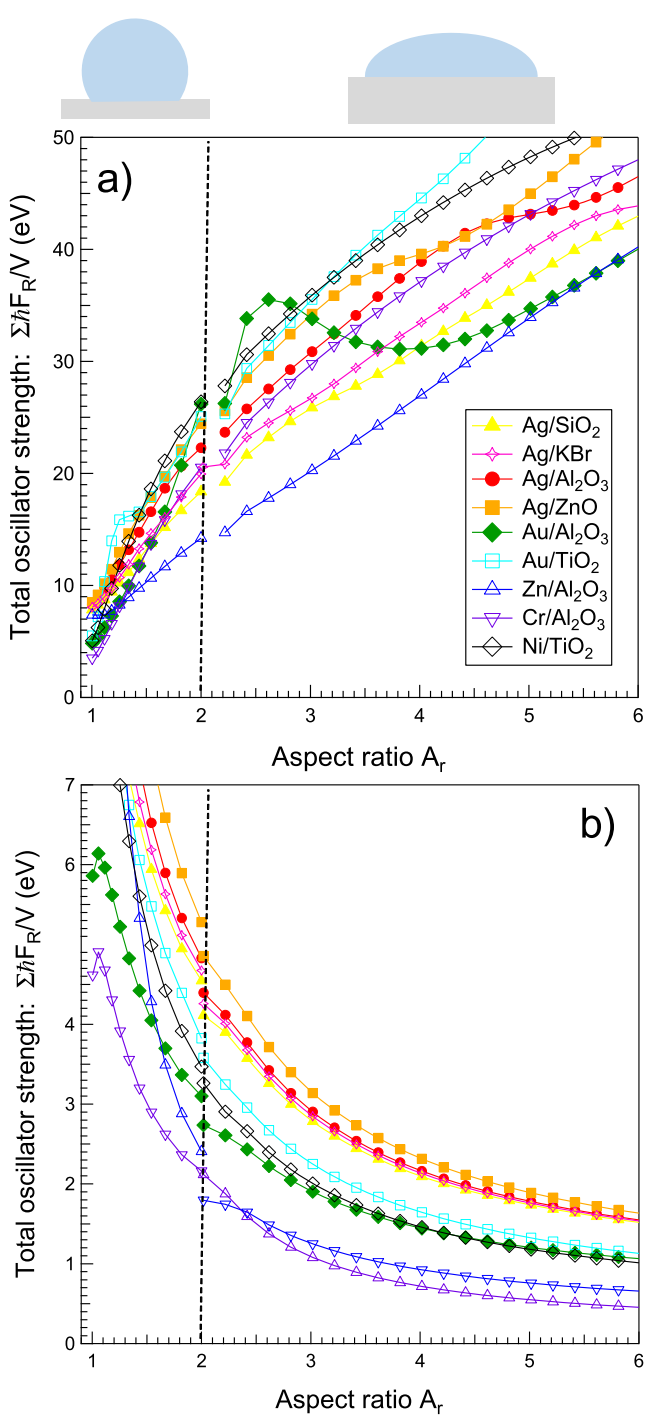

Figure 6. Oscillator strengths normalized to particle volume for isolated metal particles on dielectric substrates: (a) $\Sigma F_{R, \|} / V$ and (b) $\Sigma F_{R, \perp} / V$. Values are obtained by integration of the calculated $\operatorname{Im}[\alpha(\omega)](M=16)$ over $1.5-5 \mathrm{eV}$. Tabulated dielectric functions have been used for the indicated systems (Section SI). Only particles with $A_{r}>1$ have been considered (see top drawings) in the form of (i) truncated sphere ranging from full sphere $\left(t_{r}=1 ; A_{r}=1\right)$ to hemisphere $\left(t_{r}=0 ; A_{r}=2\right)$ and (ii) hemispheroid $\left(t_{r}=0 ; A_{r}>2\right)$.

particles that have been explored herein, involving either full or truncated objects, isolated, or in assembly, while dielectric properties are represented by means of either Drude model or tabulated functions. As these models are representative of all the approaches commonly used to describe the optical response of supported nanoparticles, the above conclusions are therefore very general. In the present context, Figure 6 is used as a chart to estimate aspect ratios from the values of the integrated oscillator strengths that are obtained directly from SDRS measurements, as it is shown in the next section.

3.4. The U-Shaped Oscillator Strength Curves during Growth. Combining the s-polarized differential reflectivity formula in the long-wavelength approximation (eq 2) in the case of a non-absorbing substrate, the expression of interface susceptibilities (eq 4), and the spectral representation of the polarizability (eq 8), it appears that the integrated signal in spolarization $\mathcal{A}_{s}(t)$ defined as follows: 


$$
\begin{gathered}
\mathcal{A}_{s}(t)=\frac{c}{4 n_{a} \cos \theta_{0} t} \int_{0}^{\infty} \frac{1}{\varepsilon_{s}(\omega)-\varepsilon_{a}} \frac{\Delta R_{s}}{R_{s}}(\omega) \frac{\mathrm{d} \omega}{\omega} \\
=\frac{1}{t} \sum_{i} \rho(t)\left\langle F_{i, \|}(t)\right\rangle=\sum_{i} \frac{\left\langle F_{i, \|}(t)\right\rangle}{\langle V(t)\rangle}
\end{gathered}
$$

is proportional to the sum of the integral of damped oscillators, i.e., to their oscillator strengths. $t=\rho(t)\langle V(t)\rangle$ stands here for the average film thickness (eq 5). As shown above, particleparticle interactions result mainly in shifts of resonance frequencies with rather a small impact on the oscillator strengths (Figure 4). Thereby, from previous conclusions (Figures 5 and 6), $\mathcal{A}_{s}(t)$ is assumed to scale directly with the average aspect ratio $\left\langle A_{r}\right\rangle$, which gives insights on the changes in wetting independently of the evolution of the particle size or density.

Equation 9 assumes a non-absorbing substrate, although, in the general case, ${ }^{45}$ eq 3 involves the real and imaginary parts of the substrate surface loss function, i.e.

$$
\begin{aligned}
\frac{\Delta R_{s}}{R_{s}} & \propto \operatorname{Im}\left[\frac{\gamma}{\varepsilon_{s}-\varepsilon_{a}}\right] \\
& =\operatorname{Re}\left[\frac{1}{\varepsilon_{s}-\varepsilon_{a}}\right] \operatorname{Im}[\gamma]-\operatorname{Im}\left[\frac{1}{\varepsilon_{s}-\varepsilon_{a}}\right] \operatorname{Re}[\gamma]
\end{aligned}
$$

Nevertheless, on the one hand, $\operatorname{Im}\left[\frac{1}{\varepsilon_{s}-\varepsilon_{a}}\right]$ is negligible within the band gap of the substrate (Figure S3), and on the other hand, the real part of the oscillator-like behavior of $\gamma$ will to a great extent cancel out the contribution of the second term upon integration. Simulations for $\mathrm{Ag} / \mathrm{ZnO}, \mathrm{Au} / \mathrm{TiO}_{2}$, and $\mathrm{Ni}$ / $\mathrm{TiO}_{2}$ showed that, for $1.5 \lesssim A_{r}$, the error is well below $10 \%$ in the case of integration within the $1.5-5 \mathrm{eV}$ range (see Figure S7).

Therefore, a qualitative hint on the geometry of growing particles can be obtained without simulations directly from integration of the SDRS signal in s-polarization (eq 9). This approach was applied to the growth of metal thin films studied herein (Figure 2). The integration was performed on a $1.5-5$ $\mathrm{eV}$ window similar to that used in simulations shown in Figure 6. Despite drastically different dielectric functions (Figures S1 and S2) and resonance frequencies (Figure 2), all the integrated signals $\mathcal{A}_{s}(t)$ exhibit a strikingly similar U-shaped profile with mean film thickness (Figure 7). According to the above analysis, this trend mainly stems from changes in aspect ratios and therefore in wetting.

The decrease in $\mathcal{A}_{s}(t)$ upon increasing the thickness at the beginning of the growth deserves attention. It is at odds with changes expected from an increase in density or coverage (Figure 4). Although happening in the submonolayer range, it occurs at too high a coverage to relate to the transition from the nucleation to growth regime. In addition, the possibility of a lower sticking coefficient in the early stages of metal/ dielectric growth should even induce a further increase in the oscillator strength since SDRS integration is normalized to thickness. A likely explanation of the first branch of the Ushaped profile is a change of equilibrium shape due to epitaxial and/or surface stresses. Indeed, as previously predicted by the generalized WKW theorem including epitaxial stress effects through elasticity theory ${ }^{9,15}$ and seen in atomistic simulations, $^{18,19,67}$ strain always acts against wetting. In epitaxial

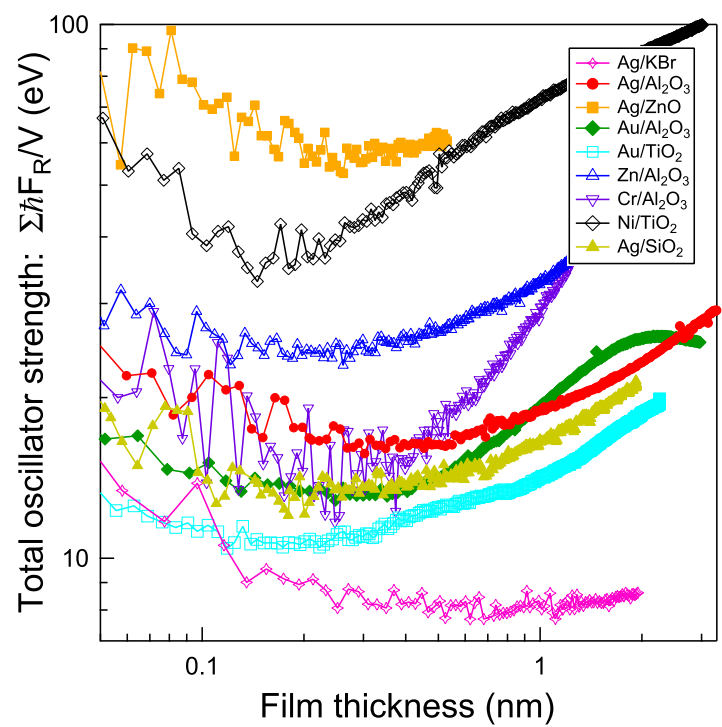

Figure 7. Evolution of the normalized total oscillator strength of modes parallel to the substrate, obtained from the integration of $\mathrm{s}$ polarization SDRS signal during the growth of various metals on dielectric substrates. Integration is performed over $1.5-5 \mathrm{eV}$. The analysis is restricted to a thickness range in which the SDRS signal affords a reliable integration. Growths are performed at room temperature, except for $\mathrm{Zn} / \mathrm{Al}_{2} \mathrm{O}_{3}(100 \mathrm{~K})$.

systems where a favorable adsorption site for the metal exists, the strain energy accumulates until the introduction of the first interfacial dislocation at which the aspect ratio reaches a minimum. ${ }^{9,17-19,67}$ Stress develops again up to the appearance of the next dislocation and so on, until the formation of larger and flatter clusters that tend to equilibrium with profiles ruled by the stress-free WKW theorem., ${ }^{9,15}$ However, the initial flattening of particles not only stems from epitaxy but also from surface stress since the same behavior is encountered in the case of amorphous substrate (Figure $7 ; \mathrm{Ag} / \mathrm{SiO}_{2}$ ). Such a stress-related interpretation perfectly matches the quantitative thorough studies of the $\mathrm{Ag} / \mathrm{Al}_{2} \mathrm{O}_{3}(0001)$ growth. ${ }^{19,20}$ The adhesion energy in this system follows a similar U-shaped profile as a function of cluster size and therefore film thickness. $^{19,20}$ The initial decrease in $\mathrm{Ag} / \mathrm{Al}_{2} \mathrm{O}_{3}(0001)$ adhesion energy that occurs within the thickness range below $t=0.5 \mathrm{~nm}$, i.e., below $D=3-5 \mathrm{~nm}$ in size, ${ }^{19,20}$ corresponds to the growth at fixed density ${ }^{20}$ of objects whose energetics is strongly affected by epitaxial stress. ${ }^{19}$ The first misfit dislocation at $\mathrm{Ag} / \mathrm{Al}_{2} \mathrm{O}_{3}(0001)^{19}$ occurs at a film thickness of about $t=0.5 \mathrm{~nm}$, which coincides with the minimum of its U-shaped adhesion energy curve. In the final stage that features the second branch of the " $U$ ", the increase in adhesion energy up to a value corresponding to the WKW profile is observed at a mean film thickness of $t>2-3 \mathrm{~nm}^{20}$ corresponding to clusters larger than $10 \mathrm{~nm}$ in size $\mathrm{e}^{20}$ for which the proportion of surface atoms is negligible. Unambiguous proofs of the thermodynamic origin of the phenomenon are provided by both the similar behavior of $\mathrm{Ag}$ films grown at various temperatures between 300 and $675 \mathrm{~K}^{20}$ and atomistic equilibrium simulations. ${ }^{19}$

Nevertheless, depending on atomic mobility, the observed late increase in $\mathcal{A}_{s}(t)$ with mean film thickness, i.e., the second branch of the U-shaped curves, can be also assigned to incomplete coalescence, i.e., the so-called elongation transition. $^{6,22-25}$ This role of kinetics is confirmed by the faster 
Table 1. Adhesion Energy $E_{a d h}$ and Aspect Ratio $A_{r, e}$ Obtained from the Minimum of the U-Shaped Curve of the Total Oscillator Strength $\Sigma F_{R, \|} / V$ (Figure 7) and from the Chart of Figure 6 for the Metal/Dielectric Interfaces under Study ${ }^{a}$

\begin{tabular}{|c|c|c|c|c|c|c|c|c|}
\hline interface & $T_{h}$ & $\Sigma F_{R, \|} / V(\mathrm{eV})$ & $A_{r, e}$ & $\gamma_{M}\left(\mathrm{~J} \cdot \mathrm{m}^{-2}\right)$ & $E_{a d h}\left(\mathrm{~J} \cdot \mathrm{m}^{-2}\right)$ & $E_{a d h}^{l i t}\left(\mathrm{~J} \cdot \mathrm{m}^{-2}\right)$ & technique & reference \\
\hline $\mathrm{Ag} / \mathrm{Al}_{2} \mathrm{O}_{3}$ & 0.24 & $16.5 \pm 0.4$ & $1.55 \pm 0.03$ & 1.25 & $0.90 \pm 0.07$ & $0.3 ; 0.2-0.9$ & SD; SDRS & 73,74 19, 20; \\
\hline $\mathrm{Ag} / \mathrm{SiO}_{2}$ & 0.24 & $13.7 \pm 0.6$ & $1.54 \pm 0.07$ & 1.25 & $0.90 \pm 0.15$ & 0.17 & $\mathrm{SD}$ & 75 \\
\hline $\mathrm{Ag} / \mathrm{ZnO}$ & 0.24 & $57 \pm 3$ & $7.2 \pm 0.4$ & 1.25 & $2.30 \pm 0.04$ & 1.58 & GISAXS & 76 \\
\hline $\mathrm{Ag} / \mathrm{KBr}$ & 0.24 & $8.2 \pm 0.3$ & $1.00 \pm 0.03$ & 1.25 & $0.0 \pm 0.2$ & - & & \\
\hline $\mathrm{Au} / \mathrm{Al}_{2} \mathrm{O}_{3}$ & 0.22 & $13.4 \pm 0.3$ & $1.54 \pm 0.03$ & 1.50 & $1.05 \pm 0.08$ & 0.26 & SD & 77 \\
\hline $\mathrm{Au} / \mathrm{TiO}_{2}$ & 0.22 & $10.8 \pm 0.3$ & $1.12 \pm 0.03$ & 1.5 & $0.30 \pm 0.15$ & $0.35-0.8$ & GISAXS & 70 \\
\hline $\mathrm{Zn} / \mathrm{Al}_{2} \mathrm{O}_{3}$ & 0.15 & $22 \pm 1$ & $3.4 \pm 0.2$ & 0.54 & $0.80 \pm 0.04$ & 0.75 & SDRS & 35 \\
\hline $\mathrm{Cr} / \mathrm{Al}_{2} \mathrm{O}_{3}$ & 0.14 & $15 \pm 2$ & $1.7 \pm 0.2$ & 2.3 & $1.9 \pm 0.8$ & 2.05 & $\mathrm{SD}$ & 8 \\
\hline $\mathrm{Ni} / \mathrm{TiO}_{2}$ & 0.17 & $38 \pm 3$ & $3.2 \pm 0.2$ & 2.45 & $3.5 \pm 0.3$ & & & \\
\hline
\end{tabular}

${ }^{a}$ Error bars represent the fluctuations of experimental points. $E_{a d h}$ are compared to values $E_{a d h}^{l i t}$ of the indicated literature references. $T_{h}$ is the homologous temperature (growth/melting temperatures). SD, SDRS, and GISAXS stand for sessile molten drop, optical reflectivity, and X-ray scattering measurements of $E_{a d h}^{\text {lit }}$.

increase in $\mathcal{A}_{s}(t)$ for $\mathrm{Zn} / \mathrm{Al}_{2} \mathrm{O}_{3}, \mathrm{Cr} / \mathrm{Al}_{2} \mathrm{O}_{3}$, and $\mathrm{Ni} / \mathrm{TiO}_{2}$ compared to all other systems, in line with their corresponding lower homologous temperature $T_{h}$ (see Table 1 ). In the context of structure zone model during continuous film formation, ${ }^{68,69} T_{h}$, defined as the ratio of growth temperature to melting point, usually helps to pinpoint low diffuse metals, the growth of which is prone to kinetic limitations. Some explanation should be provided in the case of $\mathrm{Au} / \mathrm{TiO}_{2}(110)$, for which a somewhat different evolution of aspect ratio with film thickness was found in a previous X-ray study. ${ }^{70}$ The aspect ratio was seen to decrease upon increasing the coverage, to reach a plateau value up to $t=0.6 \mathrm{~nm}$ in agreement with Figure 7 , but to finally decrease ${ }^{70}$ at odds with the present data. However, the quoted X-ray experiment was performed on a rutile crystal heavily oxidized to remove bulk and surface defects, which may explain the observed behavior. ${ }^{71}$

To refine the explanation of the $\mathcal{A}_{s}(t)$ evolution, Figure 8 compares the results obtained for $\mathrm{Ag} / \mathrm{Al}_{2} \mathrm{O}_{3}(0001)$ and $\mathrm{Ag} /$ $\mathrm{ZnO}(0001)-\mathrm{Zn}$ at different growth temperatures. The growth of $\mathrm{Ag} / \mathrm{Al}_{2} \mathrm{O}_{3}(0001)$ at temperatures higher than the ambient does not induce a notable change in the magnitude of the minimum of $\mathcal{A}_{s}(t)$ since the particles are already close to the equilibrium shape at $300 \mathrm{~K}^{19,20,45}$ At the same time, the

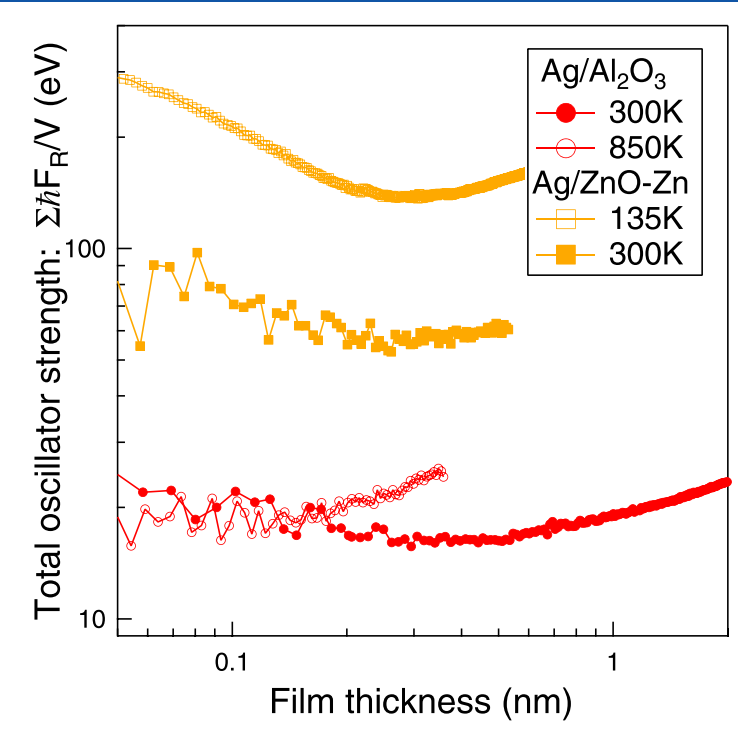

Figure 8. Same as Figure 7 but for $\mathrm{Ag} / \mathrm{Al}_{2} \mathrm{O}_{3}(0001)$ and $\mathrm{Ag} /$ $\mathrm{ZnO}(0001)-\mathrm{Zn}$ at different growth temperatures. growth of larger objects at a higher temperature results in a shift in thickness of the minimum of $\mathcal{A}_{s}(t)$. In contrast, in the $\mathrm{Ag} / \mathrm{ZnO}$ system, a flattening (or increase in $\Sigma F_{R, \|} / V$ ) is observed upon cooling down because temperature-limited diffusion hinders the recovery of the equilibrium shape either during growth of isolated islands or upon particle-particle impingement, leading to an overall shift of the curve. ${ }^{24,45}$ Therefore, despite a similar U-shaped curve for all studied systems (Figure 7), the view of size-dependent equilibrium shapes and adhesion energy ${ }^{19,20}$ gained for $\mathrm{Ag} / \mathrm{Al}_{2} \mathrm{O}_{3}(0001)$ cannot be generalized directly to all systems of supported nanoparticles analyzed herein since it would require a systematic analysis as function of flux and temperature to assess the relevance of thermodynamic equilibrium hypothesis. Nevertheless, the initial decrease in aspect ratio is likely to be driven by stress effects since it happens in a submonolayer coverage range where coalescence is limited.

3.5. Quantitative Trends in Metal/Dielectric Adhesion Energy. The chart of Figure 6 allows for the quantitative analysis of adhesion energy by assigning a value of aspect ratio $A_{r, e}$ to the minimum in $\mathcal{A}_{s}(t)$. This value offers the best compromise between stress-dependent equilibrium shape and potential kinetic effect of coalescence. Then, within the reservation that thermodynamic equilibrium is reached at this specific thickness, the corresponding adhesion energy $E_{a d h}$ can be estimated through the Young-Dupré equation for a truncated sphere:

$$
\begin{aligned}
& E_{a d h}=\gamma_{M}\left(1-t_{r}\right) \\
& t_{r}=\frac{\left(2-A_{r, e}\right)}{A_{r, e}} \text { if } A_{r, e}<2 \\
& t_{r}=\frac{-A_{r, e}^{2}+4}{A_{r, e}^{2}+4} \text { if } A_{r, e}>2
\end{aligned}
$$

Surface energies $\gamma_{M}$ of the most dense metal orientations are extracted from the compilation of ref 72 for face-centered cubic (111) [Ag, Au, Ni] and body-centered cubic (110) [Cr] and from ref 35 for hexagonal compact (0001) [Zn]. Adhesion energies for the systems under study are given in Table 1 .

The values of $E_{a d h}$ are in line with our previous analysis for $\mathrm{Ag} / \mathrm{Al}_{2} \mathrm{O}_{3},{ }^{19,20} \mathrm{Ag} / \mathrm{ZnO}(0001),{ }^{76} \mathrm{Au} / \mathrm{TiO}_{2}(110),{ }^{70}$ and $\mathrm{Zn} /$ $\mathrm{Al}_{2} \mathrm{O}_{3}(0001)^{35}$ within the inherent uncertainties of film thickness calibration with a quartz microbalance. In particular, the absolute values of $E_{a d h}$ seem to be overestimated for $\mathrm{Au}$ / 
$\mathrm{Al}_{2} \mathrm{O}_{3}$ and $\mathrm{Ag} / \mathrm{SiO}_{2}$ compared to those known from the literature. However, the very high sensitivity of the adhesion energy to the aspect ratio (as derived from the oscillator strength by means of the chart of Figure 6) for $A_{r}<2$ is likely to exacerbate the discrepancies. For example, in the case of $\mathrm{Au} / \mathrm{TiO}_{2}(110)$, a $13 \%$ increase in the aspect ratio in Table 1 doubles the adhesion energy. Furthermore, the analysis relies on the hypothesis of equilibrium shape that maybe questioned in the case of low mobility metals such as $\mathrm{Cr}$ and $\mathrm{Ni}$. Nevertheless, $E_{a d h}$ follows the general expected trends. ${ }^{75,78-83}$ In line with the larger formation energy of the corresponding oxide, i.e., the propensity to form a metal-oxide bond, $E_{a d h}$ is larger for transition metals $(\mathrm{Cr}, \mathrm{Ni})$ and $\mathrm{Zn}$ as compared to noble metals $(\mathrm{Ag}, \mathrm{Au})$ for which long-range interactions, i.e., image and van der Waals, mostly contribute to adhesion. In parallel, insulating substrates give rise to poorer adhesion compared to low band gap materials. The interaction between the metal $\mathrm{d}$ orbitals and the nonbonding oxygen $\mathrm{p}$ orbitals results in a combination of metal d-oxygen spd bonding and antibonding molecular orbitals. In parallel with the creation of favorable metal-metal bonds, their respective filling and splitting as the d-band occupancy increases leads to a decrease in adhesion from early transition metals to noble metals.

\section{CONCLUSIONS}

Changes in wetting during the growth of metal nanoparticles on dielectric substrates were probed in situ by surface differential reflectivity spectroscopy (SDRS). The search for a versatile approach for the qualitative analysis of adhesion at the metal/substrate interface led us to focus on the integrated s-polarized SDR spectra $\mathcal{A}_{s}(t)$. Upon a suitable normalization to the film thickness and the substrate dielectric function, this quantity $\mathcal{A}_{s}(t)$ is nothing else but the total oscillator strength of the plasmonic resonances parallel to the substrate surface. Numerical simulations based on a robust physical model for the optical properties of supported particles prove that $\mathcal{A}_{s}(t)$ is unambiguously linked to the aspect ratio of the growing particles and only marginally depends on particle density and particle-particle electrostatic coupling. The reason lies in the overwhelming role of a dipole-like oscillator absorption in supported truncated object.

The analysis applied to noble $(\mathrm{Ag}, \mathrm{Au})$, transition metals $(\mathrm{Cr}, \mathrm{Ni})$ and $\mathrm{Zn}$ growing on wide band gap $\left(\mathrm{Al}_{2} \mathrm{O}_{3}, \mathrm{SiO}_{2}, \mathrm{KBr}\right)$ and some semiconductor $\left(\mathrm{TiO}_{2}, \mathrm{ZnO}\right)$ substrates shows a generic U-shaped evolution of the total oscillator strength with the mean film thickness. The latter is assigned to variations of aspect ratio of nanoparticles during the growth and therefore, through the Young-Dupré equation, to changes in adhesion energy. At the onset of the film formation, dewetting with increasing thickness (the first branch of the "U") was ascribed to a size-dependent combination of interface and surface stresses. At a higher thickness, the second branch of the " $U$ " is dominated either by diffusion-limited coalescence or by the formation of large clusters ruled by the stress-free WKW law, depending on whether temperature-induced wetting changes are observed or not in the conditions of film growth. Finally, the adhesion energies deduced from the aspect ratios as determined from the minimum of the " $U$ " compare well with expected trends between materials and with literature values. Consequently, it is possible to safely conclude that, in the Volmer-Weber growth regime, particles undergo significant changes in adhesion as a function of size during growth. Both the proposed optical approach to derive aspect ratio of growing particles and the universal behavior of their energetics that has not been evidenced so far are of interest in various fundamental and applicative fields such heterogeneous catalysis and thin film growth.

\section{ASSOCIATED CONTENT}

\section{Supporting Information}

The Supporting Information is available free of charge at https://pubs.acs.org/doi/10.1021/acsanm.0c02656.

Dielectric functions of materials, oscillator characteristics of spheroid resonances, renormalized polarizabilities in assemblies of supported particles, spectral representation of truncated particle polarizabilities and absorption modes, absorbing and dispersive substrate (PDF)

\section{AUTHOR INFORMATION}

\section{Corresponding Author}

Rémi Lazzari - CNRS, Sorbonne Université, Institut des NanoSciences de Paris, UMR 7588, F-75005 Paris, France; ○ orcid.org/0000-0003-2354-1953; Email: remi.lazzari@ insp.jussieu.fr

\section{Authors}

Jacques Jupille - CNRS, Sorbonne Université, Institut des NanoSciences de Paris, UMR 7588, F-75005 Paris, France

Rémi Cavallotti - CNRS, Sorbonne Université, Institut des NanoSciences de Paris, UMR 7588, F-75005 Paris, France

Ekaterina Chernysheva - CNRS, Sorbonne Université, Institut des NanoSciences de Paris, UMR 7588, F-75005 Paris, France; Surface du Verre et Interfaces, UMR 125 CNRS/Saint-Gobain Recherche, F-93303 Aubervilliers, France

Sebastián Castilla - CNRS, Sorbonne Université, Institut des NanoSciences de Paris, UMR 7588, F-75005 Paris, France; (1) orcid.org/0000-0002-8899-0525

Maya Messaykeh - CNRS, Sorbonne Université, Institut des NanoSciences de Paris, UMR 7588, F-75005 Paris, France

Quentin Hérault - CNRS, Sorbonne Université, Institut des NanoSciences de Paris, UMR 7588, F-75005 Paris, France; Surface du Verre et Interfaces, UMR 125 CNRS/SaintGobain Recherche, F-93303 Aubervilliers, France

Iryna Gozhyk - Surface du Verre et Interfaces, UMR 125 CNRS/Saint-Gobain Recherche, F-93303 Aubervilliers, France

Elisa Meriggio - CNRS, Sorbonne Université, Institut des NanoSciences de Paris, UMR 7588, F-75005 Paris, France

Complete contact information is available at:

https://pubs.acs.org/10.1021/acsanm.0c02656

\section{Notes}

The authors declare no competing financial interest.

\section{ACKNOWLEDGMENTS}

We thank all reviewers for their very constructive comments. R.C., E.C., and Q.H. thank ANRT (Agence Nationale de la Recherche et de la Technologie), Arcelor-Mittal Maizières Research, and Saint-Gobain Recherche for the CIFRE funding of their thesis (grants 2013/0521 and 2016/0650). M.M. and E.M. acknowledge the support of the French state fund managed by the ANR (Agence Nationale de la Recherche) within the Investissements d'Avenir program under reference 
ANR-11-IDEX-0004-02 and more specifically within the framework of the Cluster of Excellence MATISSE. R.L., I.G., and Q.H. acknowledge the support of ANR (Industrial chair FRAXOS, reference ANR-15-CHIN-0003). The SDRS setup was designed by S. Chenot (INSP, Paris).

\section{REFERENCES}

(1) Kaplan, W. D.; Chatain, D.; Wynblatt, P.; Carter, W. C. A review of wetting versus adsorption, complexions, and related phenomena: the rosetta stone of wetting. J. Mater. Sci. 2013, 48, 5681-5717.

(2) Henry, C. R. Surface studies of supported model catalysts. Surf. Sci. Rep. 1998, 31, 231-325.

(3) Roldan Cuenya, B.; Behafarid, F. Nanocatalysis: size- and shapedependent chemisorption and catalytic reactivity. Surf. Sci. Rep. 2015, $70,135-187$.

(4) Ernst, F. Metal-oxide interfaces. Mater. Sci. Eng., R: Rep. 1995, $14,97-156$.

(5) Sinnott, S. B.; Dickey, E. C. Ceramic/metal interface structures and their relationship to atomic- and meso-scale properties. Mater. Sci. Eng., R: Rep. 2003, 43, 1-59.

(6) Thompson, C. V. Solid-state dewetting of thin films. Annu. Rev. Mater. Res. 2012, 42, 399-434.

(7) Campbell, C. T.; Parker, S. C.; Starr, D. E. The effect of sizedependent nanoparticle energetics on catalyst sintering. Science 2002, 298, 811.

(8) Campbell, C. T. Ultrathin metal films and particles on oxide surfaces: structural, electronic and chemisorptive properties. Surf. Sci. Rep. 1997, 27, 1-111.

(9) Müller, P.; Kern, R. Equilibrium nano-shape changes induced by epitaxial stress (generalised Wulf-Kaishew theorem). Surf. Sci. 2000, 457, 229-253.

(10) Fu, Q; Wagner, T. Interaction of nanostructured metal overlayers with oxide surfaces. Surf. Sci. Rep. 2007, 62, 431-498.

(11) Marks, L. D.; Peng, L. Nanoparticle shape, thermodynamics and kinetics. J. Phys.: Condens. Matter 2016, 28, No. 053001.

(12) Lüth, H. Surface and Interfaces of Solids; Springer Study Edition; Springer-Verlag: Berlin Heidelberg, 1992.

(13) Kaischew, R. Sur la thermodynamique des germes cristallins. Bull. Acad., Sci. Bulg., Ser. Phys. 1951, 2, 191.

(14) Winterbottom, W. L. Equilibrium shape of a small particle in contact with a foreign substrate. Acta Metall. 1967, 15, 303-310.

(15) Müller, P.; Kern, R. Equilibrium nano-shape change induced by epitaxial stress: effect of surface stress. Appl. Surf. Sci. 2000, 164, 6871.

(16) Cabrera, N. The equilibrium of crystal surfaces. Surf. Sci. 1964, 2, 320-345.

(17) Goniakowski, J.; Mottet, C. Palladium nano-clusters on the $\mathrm{MgO}(100)$ surface: substrate-induced characteristics of morphology and atomic structure. J. Cryst. Growth 2005, 275, 29-38.

(18) Mottet, C.; Goniakowski, J. Influence of epitaxial strain on supported metal cluster shapes via atomistic simulations. J. Comput. Theor. Nanosci. 2007, 4, 326.

(19) Lazzari, R.; Goniakowski, J.; Cabailh, G.; Cavallotti, R.; Trcera, N.; Lagarde, P.; Jupille, J. Surface and epitaxial stresses on supported metal clusters. Nano Lett. 2016, 16, 2574-2579.

(20) Lazzari, R.; Jupille, J. Growth kinetics and size-dependent wetting of $\mathrm{Ag} / \alpha-\mathrm{Al}_{2} \mathrm{O}_{3}(0001)$ nanoparticles studied via the plasmonic response. Nanotechnology 2012, 23, 135707.

(21) Stankic, A.; Cortes-Huerto, R.; Crivat, N.; Demaille, D.; Goniakowski, J.; Jupille, J. Equilibrium shapes of supported silver clusters. Nanoscale 2013, 5, 2448-2453.

(22) Jeffers, G.; Dubson, M. A.; Duxbury, P. M. Island to percolation transition during growth of metal films. J. Appl. Phys. 1994, 75, 50165020.

(23) Carrey, J.; Maurice, J.-L. Transition from droplet growth to percolation: Monte Carlo simulations and an analytical model. Phys. Rev. B 2001, 63, 245408.
(24) Grachev, S.; De Grazia, M.; Barthel, E.; Søndergård, E.; Lazzari, R. Real-time monitoring of nanoparticle film growth at high deposition rate with optical spectroscopy of plasmon resonances. $J$. Phys. D: Appl. Phys. 2013, 46, 375305-375315.

(25) Lü, B.; Souqui, L.; Elofsson, V.; Sarakinos, K. Scaling of elongation transition thickness during thin-film growth on weakly interacting substrates. Appl. Phys. Lett. 2017, 111, No. 084101.

(26) Jacquet, P.; Podor, R.; Ravaux, J.; Teisseire, J.; Gozhyk, I.; Jupille, J.; Lazzari, R. Grain growth: the key to understand solid-state dewetting of silver thin films. Scr. Mater. 2016, 115, 128-132.

(27) Jacquet, P.; Podor, R.; Ravaux, J.; Lautru, J.; Teisseire, J.; Gozhyk, I.; Jupille, J.; Lazzari, R. On the solid-state dewetting of polycrystalline thin films: capillary versus grain growth approach. Acta Mater. 2018, 143, 281-290.

(28) Renaud, G.; Lazzari, R.; Revenant, C.; Barbier, A.; Noblet, M.; Ulrich, O.; Leroy, F.; Jupille, J.; Borenstzein, Y.; Henry, C.; Deville, J.; Scheurer, F.; Mane-Mane, J.; Fruchart, O. Real time in situ quantitative study of growing islands by Grazing Incidence SmallAngle X-ray Scattering. Science 2003, 300, 1416-1419.

(29) Renaud, G.; Lazzari, R.; Leroy, F. Probing surface and interface morphology with Grazing Incidence Small Angle X-ray Scattering. Surf. Sci. Rep. 2009, 64, 255-380.

(30) Noguez, C. Surface plasmons on metal nanoparticles: the influence of shape and physical environment. J. Phys. Chem. C 2007, $111,3806-3819$.

(31) Halas, N. J.; Lal, S.; Chang, W.-S.; Link, S.; Nordlander, R. Plasmons in strongly coupled metallic nanostructures. Chem. Rev. 2011, 111, 3913-3961.

(32) Bohren, C. F.; Huffman, D. R. Absorption and Scattering of Light by Small Particles; Wiley-Interscience, Ed.; John Wiley \& Sons: New York, 2008.

(33) Maier, S. A. Plasmonics: fundamental and applications; Springer Science \& Business Media: 2007.

(34) Lazzari, R.; Jupille, J. Wetting and Interfacial chemistry of metallic films on the hydroxylated $\alpha-\mathrm{Al}_{2} \mathrm{O}_{3}$ (0001) surface. Phys. Rev. B 2005, 71, No. 045409.

(35) Thi Le, H.-L.; Lazzari, R.; Goniakowski, J.; Cavallotti, R.; Chenot, S.; Noguera, C.; Jupille, J.; Koltsov, A.; Mataigne, J.-M. Tuning adhesion at metal/oxide interfaces by surface hydroxylation. J. Phys. Chem. C 2017, 121, 11464-11471.

(36) Lazzari, R.; Renaud, G.; Revenant, C.; Jupille, J.; Borensztein, Y. Adhesion of growing nanoparticles at a glance: Surface differential reflectivity spectroscopy and grazing incidence small angle X-ray scattering. Phys. Rev. B 2009, 79, 125428.

(37) Abadias, G.; Simonot, L.; Colin, J. J.; Michel, A.; Camelio, S.; Babonneau, D. Volmer-Weber growth stages of polycrystalline metal films probed by in situ and real-time optical diagnostics. Appl. Phys. Lett. 2015, 107, 183105.

(38) Lazzari, R.; Roux, S.; Simonsen, I.; Jupille, J.; Bedeaux, D.; Vlieger, J. Multipolar optical absorptions in supported metallic particles: the case of $\mathrm{Ag} / \mathrm{Al}_{2} \mathrm{O}_{3}$ (0001). Phys. Rev. B 2002, 65, 235424.

(39) Lazzari, R.; Jupille, J.; Cavallotti, R.; Simonsen, I. Model-free unraveling of supported nanoparticles plasmon resonance modes. $J$. Phys. Chem. C 2014, 118, 7032-7048.

(40) Wind, M. M.; Vlieger, J.; Bedeaux, D. The polarizability of a truncated sphere on a substrate I. Phys. A 1987, 141, 33-57.

(41) Wind, M. M.; Bobbert, P. A.; Vlieger, J.; Bedeaux, D. The polarizability of a truncated sphere on a substrate II. Phys. A 1987, 143, 164-182.

(42) Simonsen, I.; Lazzari, R.; Jupille, J.; Roux, S. Numerical modeling of the optical response of supported metallic particles. Phys. Rev. B 2000, 61, 7722-7733.

(43) Lazzari, R.; Simonsen, I.; Bedeaux, D.; Vlieger, J.; Jupille, J. Polarizability of truncated spheroidal particles supported by a substrate: model and applications. Eur. Phys. J. B 2001, 24, 267-284.

(44) Bedeaux, D.; Vlieger, J. Optical Properties of Surfaces; Imperial College Press: London, 2001, DOI: 10.1142/p232.

(45) Lazzari, R.; Jupille, J. Quantitative analysis of nanoparticle growth through plasmonics. Nanotechnology 2011, 22, 445703. 
(46) Messaykeh, M. A fundamental approach of the wetting at $\mathrm{Zn} /$ $\mathrm{Cr} / \mathrm{Al} 2 \mathrm{O} 3$ interface: the effect of a Cr buffer. Ph.D. thesis, Sorbonne University, France, 2018.

(47) Lazzari, R.; Simonsen, I.; Jupille, J. Interfacial susceptibilities in nanoplasmonics via inversion of Fresnel coefficients. Plasmonics 2014, 9, 261-272.

(48) Bedeaux, D.; Vlieger, J. A phenomenological theory of the dielectric properties of thin films. Physica 1973, 67, 55-73.

(49) Albano, A. M.; Bedeaux, D.; Vlieger, J. On the description of interfacial properties using singular densities and currents at a dividing surface. Phys. A 1979, 99, 293-304.

(50) Albano, A. M.; Bedeaux, D.; Vlieger, J. On the description of interfacial electromagnetic properties using singular fields, charge density and currents at a dividing surface. Phys. A 1980, 102, 105119.

(51) Bedeaux, D.; Koper, G. J. M.; Zeeuw, E. A. v. d.; Vlieger, J.; Wind, M. The definition and use of optical invariants for thin island films. Phys. A 1994, 207, 285-292.

(52) Haarmans, M. T.; Bedeaux, D. Optical properties of thin films up to second order in the thickness. Thin Solid Films 1995, 258, 213223.

(53) McIntyre, J. D. E.; Aspnes, D. E. Differential reflection spectroscopy of very thin surface films. Surf. Sci. 1971, 24, 417-434.

(54) Bobbert, P. A.; Vlieger, J. The polarizability of a spheroidal particle on a substrate. Phys. A 1987, 147, 115-141.

(55) Yamaguchi, T.; Yoshida, S.; Kinbara, A. Optical effect of the substrate on the anomalous absorption of aggregated silver films. Thin Solid Films 1974, 21, 173-187.

(56) Lazzari, R. Vers la matrise de la croissance des couches minces: une étude par spectroscopie optique et d'électrons. Ph.D. thesis, Université Paris XI, France, 2000

(57) Letnes, P. A.; Simonsen, I.; Mills, D. L. Substrate influence on the plasmonic response of clusters of spherical nanoparticles. Phys. Rev. B 2011, 83, No. 075426.

(58) Wind, M. M.; Bobbert, P. A.; Vlieger, J.; Bedeaux, D. The polarizability of truncated spheres and oblate spheroids on a substrate: comparison with experimental results. Thin Solid Films 1988, 57-62.

(59) Román-Velázquez, C. E.; Noguez, C.; Barrera, R. G. Substrate effects on the optical properties of spheroidal nanoparticles. Phys. Rev. B 2000, 61, 10427-10436.

(60) Lazzari, R.; Simonsen, I.; Jupille, J. Onset of charge localisation on coupling multipolar absorption modes in supported metal particles. Europhys. Lett. 2003, 61, 541-546.

(61) Prodan, E.; Radloff, C.; Halas, N. J.; Nordlander, P. A hybridization model for the plasmon response of complex nanostructures. Science 2003, 302, 419-422.

(62) Lazzari, R.; Simonsen, I. GranFilm: a software for calculating thin-layer dielectric properties and Fresnel coefficients. Thin Solid Films 2002, 419, 124-136.

(63) GranFilm can be downloaded with a user guide from: http:// www.insp.jussieu.fr/-Logiciels-.html.

(64) Palik, E. D. Handbook of Optical Constants of Solids; Academic Press: 1998; Vol. 1-3.

(65) Jellison, G. E., Jr.; Batner, L. A. Optical functions of uniaxial $\mathrm{ZnO}$ determined by generalized ellipsometry. Phys. Rev. B 1998, 58, $3586-3589$.

(66) Nash, D. J.; Sambles, J. R. Surface plasmon-polariton study of the optical dielectric function of zinc. J. Mod. Opt. 1998, 45, 25852596.

(67) Olander, J.; Lazzari, R.; Jupille, J.; Mangili, B.; Goniakowski, J.; Renaud, G. Size- and temperature-dependent epitaxy for a strong filmsubstrate mismatch: The case of $\mathrm{Pt} / \mathrm{MgO}(001)$. Phys. Rev. B 2007, 76, No. 075409.

(68) Mahieu, S.; Ghekiere, P.; Depla, D.; De Gryse, R. Biaxial alignment in sputter deposited thin films. Thin Solid Films 2006, 515, $1229-1249$
(69) Depla, D.; Braeckman, B. R. Quantitative correlation between intrinsic stress and microstructure of thin films. Thin Solid Films 2016, 604, 90-93.

(70) Lazzari, R.; Renaud, G.; Jupille, J.; Leroy, F. Self-similarity during growth of the $\mathrm{Au} / \mathrm{TiO}_{2}$ (110) model catalyst as seen by the scattering of X-rays at grazing-angle incidence. Phys. Rev. B 2007, 76, 125412 .

(71) Lopez, N.; Nørskov, J. K. Theoretical study of the $\mathrm{Au} / \mathrm{TiO}_{2}$ (110) interface. Surf. Sci. 2002, 515, 175-186.

(72) Skriver, H. L.; Rosengaard, N. M. Surface energy and work function of elemental metals. Phys. Rev. B 1992, 46, 7157-7168.

(73) Chatain, D.; Chabert, F.; Ghetta, V.; Fouletier, J. New experimental setup for wettability characterization under monitored oxygen activity: II Wettability of sapphire by silver-oxygen melts. J. Am. Ceram. Soc. 1994, 77, 197-201.

(74) Muolo, M. L.; Valenza, F.; Passerone, A.; Passerone, D. Oxygen influence on ceramics wettability by liquid metals: $\mathrm{Ag} / \alpha-\mathrm{Al}_{2} \mathrm{O}_{3}$ experiments and modelling. Mater. Sci. Eng., A 2008, 495, 153-158.

(75) Sangiorgi, R.; Muolo, M. L.; Chatain, D.; Eustathopoulos, N. Wettability and work of adhesion of nonreactive liquid metals on silica. J. Am. Ceram. Soc. 1988, 71, 742-748.

(76) Jedrecy, N.; Renaud, G.; Lazzari, R.; Jupille, J. Flat-top silver nanocrystals on the two polar faces of $\mathrm{ZnO}$ : an all angle $\mathrm{X}$-ray scattering investigation. Phys. Rev. B 2005, 72, No. 045430.

(77) Chatain, D.; Coudurier, L.; Eustathopoulos, N. Wetting and interfacial bonding in ionocovalent oxide-liquid metal systems. Rev. Phys. Appl. 1988, 23, 1055-1064.

(78) Naidich, J. V. The wettability of solids by liquid metals. Prog. Surf. Membranes Sci. 1981, 14, 353.

(79) Johnson, K. H.; Pepper, S. V. Molecular-orbital model for metal-sapphire interfacial strength. J. Appl. Phys. 1982, 53, 66346637.

(80) Stoneham, A. M. Systematics of metal-insulator interfacial energies: A new rule for wetting and strong catalyst-support interactions. Appl. Surf. Sci. 1983, 14, 249.

(81) Didier, F.; Jupille, J. Simple views on metal/oxide interfaces: contributions of the long-range interactions to the adhesion energy. J. Adhes. 1996, 58, 253-261.

(82) Goniakowski, J. Transition metals on the $\mathrm{MgO}(100)$ surface: evolution of adsorption characteristics along the $4 \mathrm{~d}$ series. Phys. Rev. $B$ 1999, 59, 11047.

(83) Hemmingson, S. L.; Campbell, C. T. Trends in adhesion energies of metal nanoparticles on oxide surfaces: Understanding support effects in catalysis and nanotechnology. ACS Nano 2017, 11, 1196-1203. 